\title{
Qualitative Analysis of Establishing Diagnosis and Management of Hypertension from Patient's Perspective in Jatinangor
}

\author{
Yulia Sofiatin, ${ }^{1}$ Nazmun Lailah, ${ }^{2}$ Trully Deti Rose Sitorus, ${ }^{3}$ Rully M. A. Roesli ${ }^{4}$ \\ ${ }^{1}$ Department of Public Health Faculty of Medicine Universitas Padjadjaran, Indonesia, \\ ${ }^{2}$ Faculty of Medicine Universitas Padjadjaran, Indonesia, ${ }^{3}$ Department of Biomedical Sciences \\ Faculty of Medicine Universitas Padjadjaran, Indonesia, ${ }^{4}$ Department of Internal Medicine Faculty \\ of Medicine Universitas Padjadjaran/Dr. Hasan Sadikin General Hospital, Bandung, Indonesia
}

\section{Abstract}

Background: Blood pressure control is necessary to prevent complications in patients with hypertension. A previous study in Jatinangor showed the failure of controlling blood pressure even with pharmacological treatment. Proper diagnosis and effective management are considered to influence the condition. This study was performed to describe the process of establishing diagnosis and management for hypertension from the patient's perspective in Jatinangor.

Methods: A phenomenological approach was used. Data were collected through semi-structured questions in focus group discussions (FGDs) led by a moderator. Participants of FGDs were patients with hypertension who had undergone antihypertensive drugs therapy based on a previous study, and were invited by the local health cadres. The study was held in two villages in Jatinangor, namely Hegarmanah and Cilayung in the period of July to August 2015. Data were presented as narration and figures.

Results: There were five and eight hypertensive participants for Hegarmanah and Cilayung villages FGD, respectively. Diagnosis of hypertension in FGD participants was not only confirmed by doctors but also other health professionals, due to various accessibilities to health care facilities. Diagnosis establishment and management of hypertension were not following the protocol. Education on pharmacological and non-pharmacological management was provided by health professionals, however still lacked details and was not reviewed on every visit, resulting in a lack of compliance.

Conclusions: Process of establishing diagnosis and management of hypertension from the patient's perspective in Jatinangor is unfortunately not following the protocol, thus may influence the blood pressure control outcome.

Keywords: Diagnosis establishment, focus group discussion, hypertension, hypertension management

\section{Introduction}

Hypertension is the main cause of cardiovascular diseases such as heart attack stroke, renal failure, and early death. ${ }^{1}$ Globally, $26.4 \%$ of the adult population have hypertension and this number is predicted to rise to $29.2 \%$ in year $2025 .^{2}$ In Indonesia ${ }^{3}$, according to the Indonesian Basic Health Research (Riset Kesehatan Dasar, Riskesdas) survey in 2013, prevalence of hypertension is $26.5 \%$. West Java is the province with the third highest prevalence of hypertension $29.4 \%{ }^{3}$ Meanwhile, Jatinangor, one of the districts in Sumedang, West Java Province, has an even higher prevalence of $37.8 \%{ }^{4}$

Based on 2007-2010 data from the National Health and Nutrition Examination
Survey (NHANES), 33\% of young adults in the United States have hypertension. ${ }^{5}$ Among all young adults with hypertension, $82 \%$ are aware of ones condition, $75 \%$ consume antihypertensive drugs but only 53\% are able to achieve a controlled blood pressure level. In addition to the results, another study by Hamzah et al. ${ }^{6}$ in Jatinangor, showed that only $35 \%$ of hypertensive patients consume medications but only $6 \%$ reach a controlled blood pressure. A previous study in Jatinangor also demonstrated that there is no correlation between correct use of drug and achievement of blood pressure control. ${ }^{7}$ The study reported that patients consuming the proper drugs might still have an uncontrolled blood pressure. However, the patients consuming improper drugs achieve a controlled blood

Correspondence: Nazmun Lailah, Faculty of Medicine, Universitas Padjadjaran, Jalan Raya Bandung-Sumedang Km.21, Jatinangor, Sumedang, Indonesia, Email: nazmun.fkunpad@gmail.com 
pressure.

Proper establishment of diagnosis and effective management are keys for achieving controlled blood pressure in patients with hypertension. There are two concepts related to patients and health professionals role. ${ }^{8}$ The accurate diagnosis is influenced by the understanding of health professionals towards the process of diagnosing hypertension, while in hypertension management the health professional plans the comprehensive treatmentand educate the patients. Afterwards, it depends on whether the patient will follow the planned program or not. The purpose of this study was to investigate the process of establishing diagnosis and management from the perspective of patients in the community.

\section{Methods}

This study was designed as a qualitative descriptive study using a phenomenological approach. Data were collected through Focus Group Discussions (FGDs). The FGD was chosen since it provided an efficient, fast, and economical method in obtaining qualitative data. It developed more socially-oriented environment so that the participants would feel free to share information and answer the questions spontaneously. ${ }^{9}$ Two FGDs were conducted in Hegarmanah and Cilayung village, Jatinangor District, representing rural and urban areas. A session of FGD with health professionals working within the area were served as a triangulation process. Data were collected in the period of July to August 2015. The ethical approval for the study was obtained from the Health Research Ethics
Committee, Faculty of Medicine, Universitas Padjadjaran Bandung, No. 589/UN6.C1.3.2/ KEPK/PN/2015.

The FGD participants were respondents from a previous study who were diagnosed with hypertension and underwent antihypertensive drugs therapy. ${ }^{7}$ Subjects were recruited using the purposive sampling method. ${ }^{10}$ The list of eligible subjects was sent to the local health cadres and they selected the subjects based on adjacent residence to ensure participants' attendance. Then, a few days prior to the FGD, the cadre informed the selected subjects to join the FGD.

Each FGD was led by a moderator who is specialized in internal medicine with experience in FGD. The FGD was guided by semi-structured questions on establishing diagnosis and management of hypertension (Figure 1). FGDs were held in Sundanese language as the majority of the respondents felt more comfortable using their local language. Before each FGD was conducted, the moderator explained the purpose of the study and requested consent to audio-record the discussion.

Transcriptions in Bahasa Indonesia were made by two authors independently, and then compared to find any essential disparities which will be reconfirmed together. The data analysis included coding, categorization, theme formation, and conclusion.

\section{Results}

All informants were patients with hypertension. There were five informants from Hegarmanah village with initial blood pressure

\section{Establishment of Hypertension}

- Who diagnosed that you had hypertension?

- What was the basis of such diagnosis?

- How was the patients' experience in the process of establishing diagnosis of hypertension?

\section{Management of Hypertension}

- How was the education given regarding the plan of hypertension management?

- How was the non-pharmacological therapy of hypertension given to the patient?

- $\quad$ Did the patients consume medication for hypertension? If yes, from whom did they get the medication?

- If the drugs was given by health professionals, how was the education about the drugs (type, dosage, frequency, side effect, etc) given?

Figure 1 Semi-structured Questions as Guideliness for Moderator 
ranging between prehypertension stage I and hypertension stage II. From Cilayung village, there were eight informants who joined FGD with initial blood pressure ranging between stage I and II hypertension, prior gestational hypertension.

Hypertension was diagnosed on the first visit in almost all of the informants, and all of them received a drug treatment on the first visit. The diagnosis of hypertension among FGD informants in Hegarmanah Village was confirmed by medical doctors, either by a private doctor, doctor or nurse at the Primary Health Center (Pusat Kesehatan Masyarakat, Puskesmas). On the other hand, in Cilayung village, midwives and mantri (licensed nurses who are accustomed to prescribe drugs in regions with lack of doctors) were also involved in hypertension diagnosis.

One of the informants was diagnosed with gestational hypertension without prepregnancy blood pressure information by a midwife. Midwives in Cilayung Village provide health care privately (at home), while at the integrated health post they mainly focus on maternal and child care (Pos Pelayanan Terpandu, Posyandu), as well as at the Puskesmas Pembantu. The mantri is a wellknown nurse in Cilayung village who works in hospital and practice privately at home.

The variation of health professionals who diagnose hypertension are due to different accessibility of health care facilities between the two villages (Figure 2). Hegarmanah Village is located near the main road with a strategic access to health care facilities including Puskesmas as well as private doctors. Meanwhile, Cilayung village's location is far from Jatinangor's main road with the nearest available health care facilities only midwives and Puskesmas Pembantu, and without the availability of public transportation. The best so-called public transportation was ojek (motorcycle taxi), the cost was IDR 20,000.00 one way to and from Puskesmas. This situation inhibited informants from coming to Puskesmas to collect drugs provided through the Universal Health Coverage Uaminan Kesehatan Nasional, JKN). Health professionals confirmed the shortage of medical doctors in the area, thus the task of diagnosing and treating wase shifted to the nurse. Some nurses provided private medical practice in their neighbourhood.

Awareness among informants were low, some of them were diagnosed as hypertensive after their admission to hospital due to complications. The fact that hypertension is not always symptomatic resulted in a lack of awareness to get their blood pressure checked or managed causes complications that need urgent measures including hospitalization.

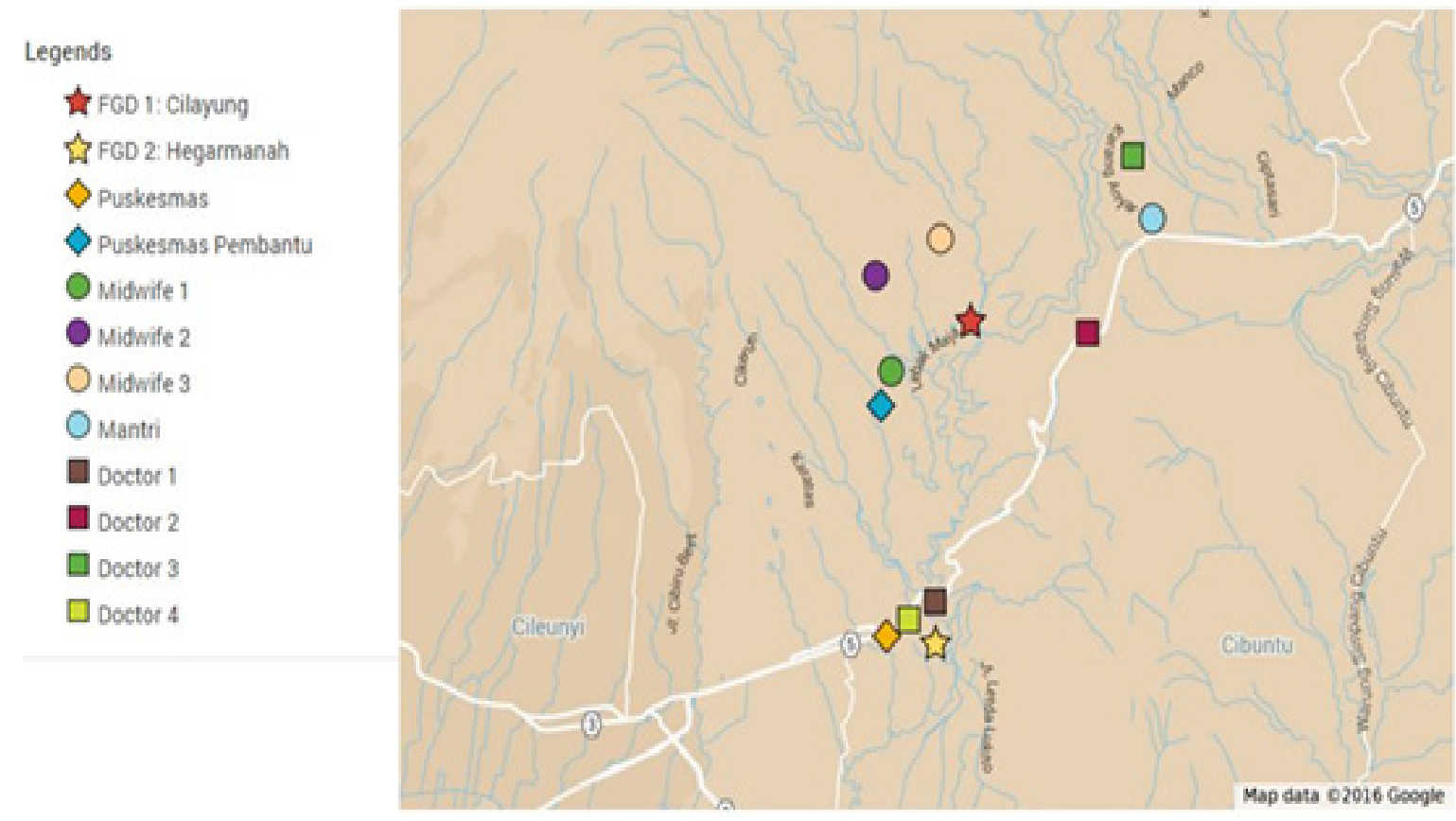

Figure 2 Map of Health Care Facilities in the Study Area (scale:1:106) 
Despite the low awareness, there is a blood pressure check peddler who offers blood pressure measurement house to house. However, a FGD informant remarked that they no longer trusted the peddler as he was also selling drugs and herbal medications so that the result mentioned was often higher than the actual blood pressure. Health professionals confirmed the presence of the peddlers, and their practice was institutionally prohibited through the village officer's monitoring.

All FGD informants were treated with drugs on the first meeting without any lifestyle modification education. The duration of drug treatment varied. Informants from Hegarmanah received medication for a week, and were recommended to control afterwards. Informants from Cilayung received medication for three days and were not recommended to control. Some of the informants received medicines for a longer period. This condition was confirmed. The reasons included the concern that those patients would not follow the life-style modification, nor return to the health facility after they felt better.

Education on the antihypertensive drugs given was still limited to explanations about the type and frequency. An informant admitted that the education provided by the doctor during the next visit was not different from the first visit with the addition to regularly take the drugs. Most of the informants could not recall what the doctor had suggested regarding the medicine.

All informants admitted to receive education on non-pharmacological management involving lifestyle modifications such as reducing salty foods consumption including salted fish, and fatty foods, increasing physical activity as well as avoiding excessive stress. Fruit and vegetables consumption was also advised; however, the term fruit was falsely assumed to 'imported' fruits they had to buy, they did not consider the local fruit grown around their living area, such as papaya, will give the same benefit to their health. Health profesionals admitted of delivering education; however they also doubted their own ability in communicating the right messages.

The notion that hypertension generally does not generate symptoms has also not been fully understood by FGD participants. Almost all participants believed that headache and dizziness were the signs that they had high blood pressure. Thus, if they did not feel as such, they did not need to consume antihypertensive drugs. Moreover, they considered that their blood pressure would always be constant; when their blood pressure increased during treatment they thought that the drug was a failure. Unfortunatelly, the similar concepts were also embraced by some health professionals. Therefore, education from health professionals on factors that raise blood pressure was needed; however education for health profesionals was also necessary.

\section{Discussions}

In this study, the process of establishing diagnosis of hypertension according to patients' experience in Jatinangor was still not following the protocol. Patients diagnosed with hypertension received antihypertensive drugs on the first visit, despite the stage of their hypertension condition. According to the Canadian Hypertension Education Program Recommendation 2015 which was adopted by the Indonesian Society of Hypertension (InaSH), hypertension should be established within several visits, except in the cases of hypertensive urgency and emergency. ${ }^{11}$ This condition will lead to overdiagnosis of hypertension and overtreatment which further leads to high cost.

General practitioners are authorized for diagnosing hypertension as well as providing full treatment for those without complications. With the shortage of GP, these tasks are shifted to nurses and, midwives who are licensed to particular regions. This arrangement will help the community to get closer to health services. However they should have additional and updated competence, and even the GPs need the update. A study on hypertension management from the general practitioner's perspective was previously conducted by Howes et al. ${ }^{12}$ through FGD. This study showed that the doctors need consistent and accurate measurement, and reading of patients' blood pressure as well as skill improvement, especially regarding the diagnostic guideline and management of hypertension.

The provider's adherence in following available clinical guidelines is an obstacle in achieving controlled blood pressure level in patients with hypertension. ${ }^{8}$ One of the reasons stated by the providers is the difficulty in the health system, which is insufficient time for patient consultations, making it hard for them to observe the diagnostic and management guideline for hypertension. Moreover, the ability to follow current information development is also a hindrance as well as their own disbelief towards available 
guidelines, whether it would yield better results or the opposite. ${ }^{8}$ In the era of universal coverage, when drugs are provided by the government, the protocol of drug dispensing, especially to residents of remote areas should be re-arranged to increase the compliance.

The phenomenon occurring in Cilayung village showed the lack of health care facilities in the region, which then caused a lack of awareness on hypertension. This was previously proven by a participant who developed an organ dysfunction due to high blood pressure without being detected with high blood pressure. This lack of awareness on hypertension as in developing countries, which is probably caused by poor accessibility of healthcare facilities was also shown in another study. ${ }^{13}$

Management of hypertension is divided to pharmacological and non-pharmacological therapy. The principle of hypertension management is to primarily encourage a lifestyle modification, afterwards if the controlled blood pressure level of $<140 / 90$ or $<130 / 80 \mathrm{mmHg}$ in patients with diabetes or renal disease is not achieved, then the administration of antihypertensive drugs can carried out. There is an exception in the treatment of hypertensive crisis, in which the administration of antihypertensive drugs to the patient should be carried out right away.

A lack of adherence to the protocols, especially on the administration of antihypertensive drugs before diagnosis of hypertension was confirmed and unsuitable competency for establishing diagnosis might be the answers for the low relationship between correct drugs consumption with achievement of controlled blood pressure in hypertensive patients.

The use of antihypertensive drugs on patients with high blood pressure may decrease the risk for stroke and other cardiovascular disease. ${ }^{14}$ the administration of antihypertensive drugs by health professionals for the FGD participants was still not following the protocol. According to the ESH-ESC Guidelines 2013, stage I and II hypertensive patients should be encouraged to first modify their lifestyle for a month and a week, afterwards antihypertensive drugs can be given if a controlled blood pressure level is unattainable. ${ }^{15}$ However, another research stated that administration of antihypertensive drugs to patients with risk, without considering their blood pressure, is given to patients with any grade of blood pressure. ${ }^{16}$

Education on pharmacological and non- pharmacological therapy given towards patients or patients' family is proven to increase patients' compliance. ${ }^{17} \mathrm{~A}$ study in Southwest Nigeria ${ }^{18}$ using FGD showed that one factor mentioned by the participant as the cause of low compliance to hypertension therapy is the low level of knowledge regarding hypertension itself. For instance, participants assume if there is no symptom felt, then it is not necessary to consume antihypertensive medicine. ${ }^{11}$ So that, education should not be limited to hypertension management, but also includes factors influencing blood pressure and possible complications in order to raise patients' knowledge on their disease. ${ }^{1}$

The limitations of this study is that there is not one member examining the discussion transcripts to increase the reliability of the study, which is due to the limited time provided. In addition, non-verbal interactions between FGD participants failed to be well documented due to the lack of field-notes.

This study concludes that the process of establishing diagnosis and management for hypertension from patients' perspective in Jatinangor is still not following the protocols, thus may influence the blood pressure control outcome. The disconcordance of education received by patients and provided by the health profesional needs to be assessed in order to plan a better hypertension prevention program, both primary and secondary.

\section{References}

1. World Health Organization. A global brief on hypertension: silent killer, global public health crises (World Health Day 2013). Geneva: WHO; 2013.

2. Kearney PM, Whelton M, Reynolds K, Muntner P, Whelton PK, He J. Global burden of hypertension: analysis of worldwide data. Lancet. 2005;365(9455):217-23.

3. Departemen Kesehatan Republik Indonesia. Riset Kesehatan Dasar (RISKESDAS) 2013. Jakarta: Badan Penelitian dan Pengembangan Kesehatan Kementerian Kesehatan, Republik Indonesia; 2013.

4. Fihaya FY, Sofiatin Y, Ong PA, Sukandar $\mathrm{H}$, Roesli RM. Prevalence of hypertension and its complications in Jatinangor 2014. J Hypertens. 2015;33(Suppl2):34-5.

5. Go AS, Mozaffarian D, Roger VL, Benjamin EJ, Berry JD, Blaha MJ, et al. Heart disease and stroke statistics--2014 update: a report from the American Heart Association. Circulation. 2014;129(3):28-292. 
6. Hamzah NKA, Roesli RM, Sofiatin Y, Sukandar H. Awareness, treatment and control of hypertension in Jatinangor subdistrict between March-November 2014. J Hypertens. 2015;33(Suppl2):34-5.

7. Putra MRD, Sofiatin Y, Hidayat EM, Sukandar H, Roesli RM. Correlation between the correct use of drug and the achievement of blood pressure control. J Hypertens. 2015;33(Suppl2):34-5.

8. Khatib R, Schwalm J-D, Yusuf S, Haynes RB, McKee M, Khan M, et al. Patient and healthcare provider barriers to hypertension awareness, treatment and follow up: a systematic review and metaanalysis of qualitative and quantitative studies. PloS one. 2014;9(1):1-12.

9. Onwuegbuzie AJ, Dickinson WB, Leech NL, Zoran AG. A qualitative framework for collecting and analyzing data in focus group research. Int J Qual Meth. 2009;8(3):1-21.

10. Wong LP. Focus group discussion: a tool for health and medical research. Singapore Med J. 2008;49(3):256-60.

11. Daskalopoulou SS, Rabi DM, Zarnke KB, Dasgupta K, Nerenberg K, Cloutier L, et al. The 2015 Canadian Hypertension Education Program recommendations for blood pressure measurement, diagnosis, assessment of risk, prevention, and treatment of hypertension. Can J Cardiol. 2015;31(5):549-68.

12. Howes F, Hansen E, Nelson M. Management of hypertension in general practice: a qualitative needs assessment of Australian
GPs. Aust Fam Physician. 2012;41(5):31723.

13. Pereira $M$, Lunet $N$, Azevedo A, Barros H. Differences in prevalence, awareness, treatment and control of hypertension between developing and developed countries. J Hypertens. 2009;27(5):96375.

14. Mazzaglia G, Ambrosioni E, Alacqua M, Filippi A, Sessa E, Immordino V, et al. Adherence to antihypertensive medications and cardiovascular morbidity among newly diagnosed hypertensive patients. Circulation. 2009;120(16):1598605.

15. Mancia G, Fagard R, Narkiewicz K, Redon J, Zanchetti A, Böhm M, et al. 2013 ESH/ ESC Guidelines for the management of arterial hypertension. Eur Heart J. 2013:31(7):1281-357.

16. Law M, Morris J, Wald N. Use of blood pressure lowering drugs in the prevention of cardiovascular disease: meta-analysis of 147 randomised trials in the context of expectations from prospective epidemiological studies. Bmj. 2009;338(1):1-19.

17. Osterberg L, Blaschke T. Adherence to medication. $\mathrm{N}$ Engl J Med. 2005;353(5):487-97.

18. Osamor PE, Owumi BE. Factors associated with treatment compliance in hypertension in Southwest Nigeria. J Health Popul Nutr. 2011;29(6):619-28. 